\title{
Are we failing vulnerable older people with diabetes in care homes?
}
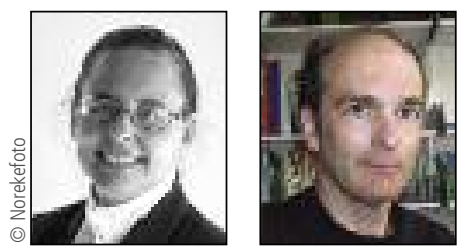

In this issue of European Diabetes Nursing we have two papers reporting on the care of older people in care homes (Hausken and Graue; Smide and Nygren). Over a quarter of residents in care homes have diabetes, ${ }^{1}$ a number that will increase as life expectancy rises. Older people with diabetes in care homes often have multiple co-morbidities, are frail and are very vulnerable. They have a high risk of avoidable hospital admission, acute complications and hypoglycaemia. Not only are these hazardous events dangerous to the older person, they can be distressing, disruptive and erode that most valuable of commodities in older age: quality of life.

The evidence suggests, however, that many older people in care homes experience poor clinical care. There is often a lack of consideration for: the nutritional needs of the older person; the need to tackle polypharmacy; the importance of preventing hypoglycaemia; the management of inter-current illness; and the need to minimise diabetes symptoms such as polyuria. Older people with diabetes in care homes may also experience problems with pain, fatigue and urinary incontinence. There is often increased mental frailty in care home residents, with high levels of cognitive impairment and dementia. This mental frailty may impede the person's capacity to self-manage their diabetes, leading to changes in the level of external support they receive with their care. In some instances, older people may be restricted in undertaking their own self-management with the routines of the institution becoming dominant over their own needs. This may create problems in the timing of medications like insulin, in blood glucose monitoring or in mealtimes. Older people have a highly elevated risk of foot complications. Foot complications affect over $20 \%$ of older people. Therefore, preventing foot problems is of vital importance in this population.

The key to solving these problems and improving the quality of life experienced by older people with diabetes in care homes is to ensure that those who are responsible for supporting them have the necessary skills to manage their diabetes effectively. Skills that should include: recognising diabetes symptoms; being aware of the selfmanagement needs of each patient; and the ability to develop and deliver an individualised care plan that will minimise risk, reduce symptoms and promote quality of life. Skills for minimising risk and preventing problems such as hypoglycaemia, foot ulcers and poly-pharmacy are essential. These skills are required at all levels of personnel: those involved in supporting the daily living needs of the person, nursing staff, the catering and nutritional staff, and the managers of the care home. Patients also require regular access to health professionals with expertise in diabetes to help construct the care plan in conjunction with the older person and their carers.

Diabetes specialist nurses can make an important contribution here, both by facilitating integrated working models and in developing care management systems to support diabetes management in care homes. Hausken and Graue through their training programme have begun to develop this contribution; we would like to advocate that the diabetes nursing community take a lead in tackling the problem of diabetes in this important and vulnerable population.

\section{Magdalena Annersten Gershater \\ Angus Forbes \\ Co-editors, EDN}

\section{Reference}

1. Sinclair AJ, et al. Prevalence of diabetes in care home residents. Diabetes Care 2001;24(6):1066-8.

\section{Get connected}

Stay one step ahead by signing up for European Diabetes Nursing e-alerts

- Find out how colleagues across Europe care for people with diabetes

- Share innovative care systems
- Discover the social and economic aspects of diabetes

- Learn about advances in management

To sign up now, simply email your name and job title to EDN@Wfley.com 